\title{
Incidence matrices of projective planes and of some regular bipartite graphs of girth 6 with few vertices *
}

\author{
C. Balbuena ${ }^{\dagger}$ \\ Departament de Matemàtica Aplicada III, \\ Universitat Politècnica de Catalunya \\ Campus Nord, Jordi Girona 1 i 3, \\ 08034Barcelona, Spain \\ m.camino.balbuena@upc.edu
}

\begin{abstract}
Let $q$ be a prime power and $r=0,1 \ldots, q-3$. Using the Latin squares obtained by multiplying each entry of the addition table of the Galois field of order $q$ by an element distinct from zero, we obtain the incidence matrices of projective planes and the incidence matrices of $(q-r)$-regular bipartite graphs of girth 6 and $q^{2}-r q-1$ vertices in each partite set. Moreover, in this work two Latin squares of order $q-1$ with entries belonging to $\{0,1, \ldots, q\}$, not necessarily the same, are defined to be quasi row-disjoint if and only if the cartesian product of any two rows contains at most one pair $(x, x)$ with $x \neq 0$. Using these quasi row-disjoint Latin squares we find $(q-1)$-regular bipartite graphs of girth 6 with $q^{2}-q-2$ vertices in each partite set. Some of these graphs have the smallest number of vertices known so far among the regular graphs with girth 6 .
\end{abstract}

Keywords: Incidence matrices, Latin squares, projective plane, girth, cages.

\section{Introduction}

Throughout this paper, only undirected simple graphs without loops or multiple edges are considered. Unless otherwise stated, we follow the book by Godsil and Royle [13] and the book by Lint and Wilson [15] for terminology and definitions.

Let $G=(V(G), E(G))$ be a graph with vertex set $V=V(G)$ and edge set $E=E(G)$. The girth of a graph $G$ is the number $g=g(G)$ of edges in a smallest cycle. The degree of a vertex $v \in V$ is the number of vertices adjacent to $v$. A graph is called regular if all the vertices have the same degree. A cage is a $k$-regular graph with girth $g$ having the smallest possible number of vertices. Simply counting the number of vertices in the distance partition with respect to a

\footnotetext{
${ }^{*}$ This work has been published in SIAM J. Discrete Maths 22(4) (2008) 1351-1363

${ }^{\dagger}$ This research was supported by the Ministry of Education and Science, Spain, and the European Regional Development Fund (ERDF) under project MTM2005-08990-C02-02
} 
vertex yields a lower bound $n_{0}(k, g)$ on the number of vertices in a cage $n(k, g)$, with the precise form of the bound depending on whether $g$ is even or odd.

$$
n_{0}(k, g)= \begin{cases}1+k+k(k-1)+\ldots+k(k-1)^{(g-3) / 2} & \text { if } g \text { is odd } \\ 2\left(1+(k-1)+\ldots+(k-1)^{g / 2-1}\right) & \text { if } g \text { is even }\end{cases}
$$

Biggs [6] call excess of a $k$-regular graph $G$ the difference $|V(G)|-n_{0}(k, g)$. Cages have been studied intensely since they were introduced by Tutte [21] in 1947. Erdös and Sachs [10] proved the existence of a graph for any value of the regularity $k$ and the girth $g$, thus most of the work carried out has been focused on constructing a smallest one $[1,3,5,8,11,12,18,16,17,23]$. Biggs is the author of an impressive report on distinct methods for constructing cubic cages [7]. Royle [20] keeps a web-site in which all the cages known so far appear. More details about constructions on cages can be found in the survey by Wong [23] or in the survey by Holton and Sheehan [14].

It is conjectured that cages with even girth are bipartite $[19,23]$. If the vertices of a bipartite graph are ordered in such a way that the vertices of one partite set come first, then the adjacency matrix can be written in the form

$$
A=\left(\begin{array}{ll}
0 & N \\
N^{\top} & 0
\end{array}\right)
$$

An incidence graph is a bipartite graph in which the elements of one partite set are declared as lines and the elements of the other partite set are declared as points. The terminology for incidence graphs is geometric. A point and a line are said to be incident if they are adjacent, thus the submatrix $N$ of (2) is called an incidence matrix of the bipartite graph. If the number of points and the number of lines coincide, then $N$ is clearly a square matrix. An incidence matrix $N$ defines a partial plane when

- any line has at least two points, and

- two points are incident with at most one line.

Consequently, two lines of a partial plane have at most one point in common. The corresponding bipartite graph is called the incidence graph of the partial plane. Clearly the incidence graph of a partial plane has even girth $g \geq 6$.

In this work we are interested in the construction of $k$-regular bipartite graphs of girth 6 with small excess. When the degree $k=p^{n}+1$, where $q=k-1=p^{n}$ is a prime power, $(k, 6)$-cages are obtained as the incidence graphs of projective planes of order $q=k-1$. A projective plane is a partial plane satisfying the following conditions:

(i) Any two lines meet at a single point.

(ii) Any two points lie on a single line.

(iii) There exists a set of four points no three of which are collinear. 
As a consequence of these conditions, it follows that a projective plane has $q^{2}+q+1$ lines and $q^{2}+q+1$ points, thus it is said to have order $q$. The incidence graph of a projective plane is a $(q+1)$-regular bipartite graph with diameter 3 and girth six. It has $q^{2}+q+1$ vertices in each partite set, and then this graph is a $(q+1,6)$-cage because its order achieves the lower bound given in (1).

One way for obtaining a projective plane when $q$ is a prime power, is to consider the three dimensional vector space $\mathbb{F}_{q}^{3}$ over the field $\mathbb{F}_{q}$ with $q$ elements. The points are the 1-dimensional subspaces and the lines are the 2-dimensional subspaces. A point is incident with a line if the 1-dimensional is contained in the 2-dimensional subspace. It is not hard to see that there are $\left(q^{3}-1\right) /(q-1)=q^{2}+q+1$ points and the same number of lines, so this structure is a projective plane denoted by $P G(2, q)$. For more details on this construction of projective planes, see for example $[4,13,15]$.

In this work we present an alternative way to construct projective planes of order a prime power, and other partial planes whose incidence graphs are $k$-regular bipartite graphs of girth 6 with small excess. Let us recall that a Latin square of order $q$ is a $q \times q$ matrix with entries from a set of $q$ symbols such that each symbol occurs exactly once in each row and exactly once in each column. Using the Latin squares obtained by multiplying each entry of the addition table of the Galois field of order $q$ by an element distinct from zero, we obtain the incidence matrices of projective planes and the incidence matrices of $(q-r)$-regular bipartite graphs of girth 6 and $q^{2}-r q-1$ vertices in each partite set. $q$-regular bipartite graphs with $q$ a prime power and $2\left(q^{2}-1\right)$ vertices of girth 6 have recently been obtained in [1]. We also improve this result for $r=1$, finding $(q-1)$-regular bipartite graphs of girth 6 with $2\left(q^{2}-q-2\right)$ vertices. To do this we use a family of Latin squares called quasi row-disjoint, a property that will be introduced in the last section. It follows from (1) that the $(q-1)$-regular bipartite graphs constructed in this work have an excess $|V(G)|-n_{0}(q-1,6)=2(2 q-5)$. And the $(q-r)$-regular bipartite graphs for $r \geq 2$ have an excess of $|V(G)|-n_{0}(q-r, 6)=2\left(r q+q-r^{2}-r-2\right)=2(q-r)(r+1)-4$. In fact, some of these graphs have the smallest number of vertices known so far among the regular graphs with girth 6 .

\section{$2 C_{4}$-free set of matrices}

Throughout this work let $[[n]]$ denote the set of non negative integers $\{0,1, \ldots, n\}$ and $(n]]=$ $[[n]] \backslash\{0\}=\{1, \ldots, n\}$. Let $A$ be a matrix or order $\alpha \times \beta$ whose elements are subsets of $[[n]]$. Let $P_{x}(A)$ be a matrix of zeros and ones of the same order as $A$ that satisfies

$$
\left(P_{x}(A)\right)_{i j}=1 \text { if and only if } x \in A_{i j}
$$

Thus, $P_{x}(A)$ is called the position matrix of the symbol $x$ in $A$. The position matrices of all the symbols in $A$ different from zero give rise to the following $(0,1)$-matrix $\mathcal{P}(A)$ of order $\alpha \times n \beta$ called position matrix of $A$ :

$$
[\mathcal{P}(A)]=\left[P_{1}(A) \cdots P_{n}(A)\right]
$$


Let $\left\{A^{1}, A^{2}, \ldots, A^{s}\right\}$ be a family of $s$ matrices of the same number of columns whose elements are subsets of $[[n]]$. Then the $(0,1)$-matrix spanned by the position matrices of all of them

$$
\left(\begin{array}{c}
\mathcal{P}\left(A^{1}\right) \\
\mathcal{P}\left(A^{2}\right) \\
\vdots \\
\mathcal{P}\left(A^{s}\right)
\end{array}\right)=\left(\begin{array}{ccc}
P_{1}\left(A^{1}\right) & \cdots & P_{n}\left(A^{1}\right) \\
P_{1}\left(A^{2}\right) & \cdots & P_{n}\left(A^{2}\right) \\
& \vdots & \\
P_{1}\left(A^{s}\right) & \cdots & P_{n}\left(A^{s}\right)
\end{array}\right)
$$

is said to be the position matrix of the family $\mathcal{F}=\left\{A^{1}, A^{2}, \ldots, A^{s}\right\}$. The next example shows two matrices of order $2 \times 2$ whose elements are subsets of [[2]] and the position matrix of them. From now on, if there is no confusion the 1-sets will be indicated as integers.

$$
A^{1}=\left(\begin{array}{ll}
1 & 1 \\
2 & 2
\end{array}\right), A^{2}=\left(\begin{array}{cc}
\{1,2\} & 0 \\
0 & \{1,2\}
\end{array}\right),\left(\begin{array}{l}
\mathcal{P}\left(A^{1}\right) \\
\mathcal{P}\left(A^{2}\right)
\end{array}\right)=\left(\begin{array}{llll}
1 & 1 & 0 & 0 \\
0 & 0 & 1 & 1 \\
1 & 0 & 1 & 0 \\
0 & 1 & 0 & 1
\end{array}\right)
$$

As already mentioned in the introduction, our main aim is to obtain incidence matrices of bipartite $k$-regular graphs of girth 6 with small excess. Such incidence matrices shall be incidence matrices of partial planes. A basic known fact is that a $(0,1)$-matrix $N$ is the incidence matrix of a partial plane if $N$ is free of any submatrix $J_{2}$ of order $2 \times 2$ full of ones. Then, to achieve our purpose we propose the following definitions.

Definition 2.1 A family of matrices $\mathcal{F}=\left\{A^{1}, A^{2}, \ldots, A^{s}\right\}$ of the same number of columns whose elements are subsets of $[[n]]$ is said to be $C_{4}$-free if the position matrix of $\mathcal{F}$ does not contain a sub-matrix $J_{2}$ of order $2 \times 2$ full of ones.

Notice that the position matrix of $\left\{A^{1}, A^{2}\right\}$ in (4) is free of $J_{2}$, then according to Definition 2.1 , the set $\left\{A^{1}, A^{2}\right\}$ is $C_{4}$-free.

Let $I_{n}$ be the identity matrix and denote by $S I_{n}$ the matrix obtained from $I_{n}$ replacing each one with a subset $S$ of $(n]]$. It is also clear that the position matrix of $S I_{n}$ is the incidence matrix of a partial plane consisting of $n$ parallel lines. The above example (4) is a particular case of the following helpful remark.

Remark 2.1 Let $A$ be any $C_{4}$-free matrix of $n$ columns whose entries are 1-sets of [[n]] and $S$ a subset of $(n]]$. Then the set $\left\{A, S I_{n}\right\}$ is $C_{4}$-free.

Proof: Note that $P_{x}\left(S I_{n}\right)=I_{n}$ for each symbol $x$ in $S$. Therefore, the position matrix of the set $\left\{A, S I_{n}\right\}$ is $J_{2}$-free because the entries of $A$ are 1 -sets.

Our immediate concern is to obtain some method for constructing families of $C_{4}$-free matrices in such a way the position matrix of all of them will be a $J_{2}$-free $(0,1)$-matrix. 
Let us recall that two Latin squares $L^{1}$ and $L^{2}$ of order $n$ with $n$ distinct symbols are orthogonal if all the $n^{2}$ pairs of $L^{1} \times L^{2}$ are distinct. The $(1,3)$ conjugate of a Latin square $L$ of order $n$ on $n$ symbols denoted by $(1,3) L$ is defined by $((1,3) L)_{i j}=a$ if and only if $(L)_{a j}=i$; that is to say, the $(1,3)$ conjugate of $L$ is obtained by interchanging the roles of rows and symbols.

Theorem 2.1 Let $L$ and $Q$ be two Latin squares of order $n$ both with entries from ( $n]$ ]. If $L$ and $Q$ are orthogonal, then their (1,3) conjugates form a family $\{(1,3) L,(1,3) Q\} C_{4}$-free.

Proof: Suppose $(L)_{a b}=i,(L)_{c d}=i$ and $(Q)_{a b}=k$. Since $L$ and $Q$ are orthogonal, then the row $x$ for which $(Q)_{x d}=k$ is never equals $c$. The $(1,3)$ conjugates are $((1,3) L)_{i b}=a$, $((1,3) L)_{i d}=c,((1,3) Q)_{k b}=a$ and $((1,3) Q)_{k d}=x$, with $x \neq c$. Therefore, the position matrix of $\{(1,3) L,(1,3) Q\}$ is $J_{2}$-free because

$$
\left(\begin{array}{cc}
\left(P_{a}((1,3) L)\right)_{i b} & \left(P_{c}((1,3) L)\right)_{i d} \\
\left(P_{a}((1,3) Q)\right)_{k b} & \left(P_{c}((1,3) Q)\right)_{k d}
\end{array}\right)=\left(\begin{array}{cc}
1 & 1 \\
1 & 0
\end{array}\right) .
$$

Let $q$ be a prime power and $\mathbb{F}_{q}$ the Galois field of order $q$. A set of $q-1$ mutually orthogonal Latin squares (MOLS) of order $q$ with entries $[[q-1]]$ are obtained according to the following rule (see [15]):

$$
\left(L^{u}\right)_{i j}=u i+j, u, i, j \in \mathbb{F}_{q}, u \neq 0 .
$$

Let $L_{q}^{u}$ be the matrix as defined in (5) but the symbol zero is replaced with $q$. Clearly, the set $\left\{L_{q}^{u}: u \in \mathbb{F}_{q} \backslash\{0\}\right\}$ is a set of $q-1$ mutually orthogonal Latin squares (MOLS) of order $q$ with entries in $(q]]$. As a consequence of Theorem 2.1 we obtain the following result.

Theorem 2.2 Let $q$ be a prime power and $\mathbb{F}_{q}$ the Galois field of order $q$. Then the following assertions hold:

(i) For each $u \in \mathbb{F}_{q}, u \neq 0$ define the matrix $\Sigma_{q}^{u}$ by $\left(\Sigma_{q}^{u}\right)_{i j}=u(i+j)$ for $i, j \in \mathbb{F}_{q}, i \neq-j$, and $q$ otherwise. Then $\left\{\Sigma_{q}^{u}: u \in \mathbb{F}_{q}, u \neq 0\right\}$ is $C_{4}$-free.

(ii) For each $u \in \mathbb{F}_{q}, u \neq 0$ define the matrix $\Upsilon_{q}^{u}$ by $\left(\Upsilon^{u}\right)_{i j}=u(i+j)^{-1}$ for $i, j \in \mathbb{F}_{q}, i \neq-j$, and $q$ otherwise. Then $\left\{\Upsilon_{q}^{u}: u \in \mathbb{F}_{q}, u \neq 0\right\}$ is $C_{4}$-free.

(iii) Let $\Sigma^{u}, \Upsilon^{u}$ be the Latin squares obtained from $\Sigma_{q}^{u}$ and $\Upsilon_{q}^{u}$ respectively, by replacing symbol $q$ with zero. If $M$ is the position matrix of $\left\{\Sigma^{u}: u \in \mathbb{F}_{q}, u \neq 0\right\}$, then the transpose of $M$ is the position matrix of $\left\{\Upsilon^{u}: u \in \mathbb{F}_{q}, u \neq 0\right\}$. 
Proof: $(i)$ Let $a=u(i+j)$ for $u, i, j \in \mathbb{F}_{q}, u \neq 0, i \neq-j$. Then the entry $(a, j)$ of the $(1,3)$ conjugate of the Latin square $\Sigma^{u}$ is $\left((1,3) \Sigma^{u}\right)_{a j}=i=u^{-1} a-j$; and for $i=-j$ we have $\left((1,3) \Sigma^{u}\right)_{q j}=-j$ because $\left(\Sigma^{u}\right)_{-j, j}=q$. According to (5) the family $\left\{(1,3) \Sigma^{u}: u \in \mathbb{F}_{q}, u \neq 0\right\}$ is a set of $q-1$ MOLS of order $q$ with entries from $(q]$. Hence by Theorem 2.1 we obtain that $\left\{\Sigma^{u}: u \in \mathbb{F}_{q}, u \neq 0\right\}$ is $C_{4}$-free. Thus point $(i)$ is valid.

(ii) Let $b=u(i+j)^{-1}$ for $u, i, j \in \mathbb{F}_{q}, u \neq 0, i \neq-j$. Then the entry $(b, j)$ of the $(1,3)$ conjugate of the Latin square $\Upsilon^{u}$ is $\left((1,3) \Upsilon^{u}\right)_{b j}=i=u b^{-1}-j$; and for $i=-j$ we have $\left((1,3) \Upsilon^{u}\right)_{q j}=-j$ because $\left(\Upsilon^{u}\right)_{-j, j}=q$. Then $\left\{\Upsilon_{q}^{u}: u \in \mathbb{F}_{q}, u \neq 0\right\}$ is a set of $q-1$ MOLS of order $q$ with entries from $(q]]$ and again by Theorem 2.1 we conclude point $(i i)$.

(iii) Let $P_{x}\left(\Sigma^{u}\right)$ be the position matrix of the element $x$ in $\Sigma^{u}$, and observe that $P_{x}\left(\Sigma^{u}\right)$ is a symmetric matrix because $\left(P_{x}\left(\Sigma^{u}\right)\right)_{i j}=1$ if and only if $u(i+j)=x, i, j, x, u \in \mathbb{F}_{q}, u, x \neq 0$. Similarly let $P_{x}\left(\Upsilon^{w}\right)$ denote the position matrix of element $x$ in $\Upsilon^{w}$, which is also symmetric. According to (3), the position matrix of $\left\{\Sigma^{u}: u \in \mathbb{F}_{q}, u \neq 0\right\}$ is

$$
M=\left(\begin{array}{ccc}
P_{1}\left(\Sigma^{1}\right) & \cdots & P_{q-1}\left(\Sigma^{1}\right) \\
\vdots & \vdots & \vdots \\
P_{1}\left(\Sigma^{q-1}\right) & \cdots & P_{q-1}\left(\Sigma^{q-1}\right)
\end{array}\right)
$$

and the position matrix of $\left\{\Upsilon^{u}: u \in \mathbb{F}_{q}, u \neq 0\right\}$ is

$$
M^{D}=\left(\begin{array}{ccc}
P_{1}\left(\Upsilon^{1}\right) & \cdots & P_{q-1}\left(\Upsilon^{1}\right) \\
\vdots & \vdots & \vdots \\
P_{1}\left(\Upsilon^{q-1}\right) & \cdots & P_{q-1}\left(\Upsilon^{q-1}\right)
\end{array}\right) .
$$

To see that $M^{D}$ is the transpose of $M$ let us show that the column $x$ of $M$ is the row $x$ of $M^{D}$, i.e. let us show that $P_{x}\left(\Sigma^{w}\right)=P_{w}\left(\Upsilon^{x}\right)$. Since $\left(P_{x}\left(\Sigma^{w}\right)\right)_{i j}=1$ if and only if $w(i+j)=x$, then $i \neq-j$ because $x \neq 0$, hence $w=x(i+j)^{-1}$ yielding $\left(P_{w}\left(\Upsilon^{x}\right)\right)_{i j}=1$. Therefore the theorem is valid.

\section{Method}

Let us call array of $r$ symbols and $n$ columns the matrix of order $r \times n$

$$
O_{r, n}=\left(\begin{array}{lll}
1 & \cdots & 1 \\
2 & \cdots & 2 \\
\vdots & \vdots & \vdots \\
r & \cdots & r
\end{array}\right)
$$

When $r=n$ the array is denoted by $O_{n}$. It is easy to see that $O_{r, n}$ is a $C_{4}$-free matrix defined on $(r]]$. Let $(k]] I_{n}$ denotes the matrix obtained from the identity matrix $I_{n}$ of order $n$ by replacing each one with the set $(k]]$. 
The following theorem is a consequence of Theorem 2.2, and gives a general method for constructing the desired $k$-regular bipartite graphs of girth 6 when $k=q, q+1$. The position matrix of $O_{q, n}$ will play an important role in what follows, and as in the above sections it will be denoted by $\left[\mathcal{P}\left(O_{q, n}\right)\right]$.

Theorem 3.1 Let $q$ be a prime power and $\mathbb{F}_{q}$ a finite Galois field of $q$ elements. Let us consider the Latin squares $\Sigma^{u}=[u(i+j)]$ with $u, i, j \in \mathbb{F}_{q}, u \neq 0$, and the Latin squares $\Sigma_{q}^{u}$ obtained from $\Sigma^{u}$ by replacing 0 with $q$. Then the following assertions hold:

(i) Assume $q \geq 3$. The position matrix of $\left.\left\{\Sigma_{q}^{1}, \Sigma_{q}^{2}, \ldots, \Sigma_{q}^{q-1},(q]\right] I_{q}\right\}$ (given by (3)) is the incidence matrix of a q-regular bipartite graph of girth 6 and $q^{2}$ vertices in each partite set.

(ii) The position matrix $A$ of $\left.\left\{\Sigma_{q}^{1}, \Sigma_{q}^{2}, \ldots, \Sigma_{q}^{q-1},(q]\right] I_{q}, O_{q}\right\}$ is the incidence matrix of an affine plane of order $q$.

Let $j_{q+1}$ be the row-matrix of order $1 \times(q+1)$ full of ones. The following $(0,1)$-matrix

\begin{tabular}{|c|c|}
\hline$A$ & $\mathcal{P}\left(O_{q+1, q}\right)^{\top}$ \\
\hline $0 \cdots 0$ & $j_{q+1}$ \\
\hline
\end{tabular}

is the incidence matrix of a projective plane of order $q$.

(iii) Let $M$ be the position matrix of $\left.\left\{\Sigma^{1}, \Sigma^{2}, \ldots, \Sigma^{q-2}, \quad(q-1]\right] I_{q}, O_{q-1, q}\right\}$. Then $\left[M \mid \mathcal{P}\left(O_{q-1, q}\right)^{\top}\right]$ is the incidence matrix of a q-regular bipartite graph of girth 6 and $q^{2}-1$ vertices in each partite set.

Proof: $(i)$ From Theorem 2.2 we know that $\left\{\Sigma_{q}^{u}: u \in \mathbb{F}_{q} \backslash\{0\}\right\}$ is $C_{4}$-free, and moreover, by Remark 2.1, we know that $\left.\{(q]] I_{q}, \Sigma_{q}^{u}\right\}$ is $C_{4}$-free. Thus it follows that $\left.\mathcal{F}=\left\{\Sigma_{q}^{1}, \Sigma_{q}^{2}, \ldots, \Sigma_{q}^{q-1},(q]\right] I_{q}\right\}$ is a $C_{4}$-free family, that is, the position matrix $N$ of $\mathcal{F}$ is free of $J_{2}$. Since each matrix of $\mathcal{F}$ has $q$ symbols in each one of its rows, then there are $q$ ones in each row of $N$. On the other hand, the position matrix $B$ of $\left\{\Sigma^{1}, \Sigma^{2}, \ldots, \Sigma^{q-1}\right\}$ is a submatrix of $N$ obtained by deleting the $q$ last rows and the $q$ last columns of $N$. From item (iii) of Theorem 2.2, it follows that the transpose $B^{\top}$ of $B$ is the position matrix of $\left\{\Upsilon^{1}, \Upsilon^{2}, \ldots, \Upsilon^{q-1}\right\}$. By the definition of the matrices $\Upsilon^{u}$ it is clear that there are $q-1$ ones in each row of $B^{\top}$. Therefore $B$ has also $q-1$ ones in each column, hence $N$ has $q$ ones in each column, thus $N$ is the incidence matrix of a $q$-regular bipartite graph of $q^{2}$ vertices in each partite set of girth 6 (notice that the number of vertices of the constructed graphs is strictly less than the lower bound given in (1) for $g=8$ ).

(ii) From Theorem 2.2 and by Remark 2.1, it follows that the position matrix $A$ of $\left.\left\{\Sigma_{q}^{1}, \Sigma_{q}^{2}, \ldots, \Sigma_{q}^{q-1},(q]\right] I_{q}, O_{q}\right\}$ is free of $J_{2}$, and clearly, there are $q$ ones in each row of $A$. By item $(i)$ we know that the position matrix of $\left.\left\{\Sigma_{q}^{1}, \Sigma_{q}^{2}, \ldots, \Sigma_{q}^{q-1},(q]\right] I_{q}\right\}$ has order $q^{2} \times q^{2}$ and $q$ ones in each row and column. Consequently, the matrix $A$ has order $\left(q^{2}+q\right) \times q^{2}$ and has $q$ ones in each row and $q+1$ ones in each column. Hence, considering the rows of $A$ as lines of a partial 
plane and the columns as points, the matrix $A$ may be seen as the incidence matrix of an affine plane of order $q$. Thus a projective plane can be obtained by adding $q+1$ points (columns) and one new line (row) containing them. The $q+1$ points correspond to the last $q+1$ columns of (6) and the new line is the last row.

(iii) The position matrix $M$ of $\left.\left\{\Sigma^{1}, \Sigma^{2}, \ldots, \Sigma^{q-2},(q-1]\right] I_{q}, O_{q-1, q}\right\}$ is a submatrix of the block $A$ of (6). Indeed, $\Sigma^{u}$ is obtained by replacing $q$ with 0 in $\Sigma_{q}^{u}$. This is equivalent to deleting in $A$ the last $q$ columns. Moreover, now matrix $\Sigma^{q-1}$ is not considered, which is equivalent to deleting in $A$ the $q$ rows corresponding to the position matrix of $\Sigma^{q-1}$. And take $O_{q-1, q}$ instead of $O_{q}$ is the same as deleting in $A$ the last row. Therefore $\left[M \mid \mathcal{P}\left(O_{q-1, q}\right)^{\top}\right]$ is a submatrix of (6) and so is free of $J_{2}$. This $(0,1)$-matrix has $q^{2}-1$ rows and $\left(q^{2}-q\right)+(q-1)=q^{2}-1$ columns and, by construction there are $q$ ones in each row and in each column. Hence, it is the incidence matrix of a $q$-regular bipartite graph of $q^{2}-1$ vertices in each partite set and girth 6 (notice that the number of vertices of the constructed graphs is strictly less than the lower bound given in (1) for $g=8$ ).

To illustrate the method contained in Theorem 3.1, let us consider the first cases $q=2,3,4$. From now on, if there is no confusion a $t$-set $\left\{x_{1}, x_{2}, \ldots, x_{t}\right\}$ will be denoted as a sequence of integers $x_{1} x_{2} \cdots x_{t}$.

Case $q=2,3$. The Latin squares provided by Theorem 2.2, and the incidence matrix of projective plane $P G(2,2)$ (called Fano plane) and the incidence matrix of projective plane $P G(2,3)$ given in $(6)$ are the following:

\begin{tabular}{|c|cc||cc|cc|ccc|}
\hline$\Sigma_{2}^{1}$ & 2 & 1 & 0 & 1 & 1 & 0 & 1 & 0 & 0 \\
& 1 & 2 & 1 & 0 & 0 & 1 & 1 & 0 & 0 \\
\hline$(2]] I_{2}$ & 12 & 0 & 1 & 0 & 1 & 0 & 0 & 1 & 0 \\
& 0 & 12 & 0 & 1 & 0 & 1 & 0 & 1 & 0 \\
\hline$O_{2}$ & 1 & 1 & 1 & 1 & 0 & 0 & 0 & 0 & 1 \\
& 2 & 2 & 0 & 0 & 1 & 1 & 0 & 0 & 1 \\
\hline & & & 0 & 0 & 0 & 0 & 1 & 1 & 1 \\
\hline
\end{tabular}

\begin{tabular}{|c|ccc||ccc|ccc|ccc|cccc|}
\hline \multirow{5}{*}{$\Sigma_{3}^{1}$} & 3 & 1 & 2 & 0 & 1 & 0 & 0 & 0 & 1 & 1 & 0 & 0 & 1 & 0 & 0 & 0 \\
& 1 & 2 & 3 & 1 & 0 & 0 & 0 & 1 & 0 & 0 & 0 & 1 & 1 & 0 & 0 & 0 \\
& 2 & 3 & 1 & 0 & 0 & 1 & 1 & 0 & 0 & 0 & 1 & 0 & 1 & 0 & 0 & 0 \\
\hline \multirow{5}{*}{$\Sigma_{3}^{2}$} & 3 & 2 & 1 & 0 & 0 & 1 & 0 & 1 & 0 & 1 & 0 & 0 & 0 & 1 & 0 & 0 \\
& 2 & 1 & 3 & 0 & 1 & 0 & 1 & 0 & 0 & 0 & 0 & 1 & 0 & 1 & 0 & 0 \\
& 1 & 3 & 2 & 1 & 0 & 0 & 0 & 0 & 1 & 0 & 1 & 0 & 0 & 1 & 0 & 0 \\
\hline \multirow{5}{*}{$(3]] I_{3}$} & 123 & 0 & 0 & 1 & 0 & 0 & 1 & 0 & 0 & 1 & 0 & 0 & 0 & 0 & 1 & 0 \\
& 0 & 123 & 0 & 0 & 1 & 0 & 0 & 1 & 0 & 0 & 1 & 0 & 0 & 0 & 1 & 0 \\
& 0 & 0 & 123 & 0 & 0 & 1 & 0 & 0 & 1 & 0 & 0 & 1 & 0 & 0 & 1 & 0 \\
\hline \multirow{3}{*}{$O_{3}$} & 1 & 1 & 1 & 1 & 1 & 1 & 0 & 0 & 0 & 0 & 0 & 0 & 0 & 0 & 0 & 1 \\
& 2 & 2 & 2 & 0 & 0 & 0 & 1 & 1 & 1 & 0 & 0 & 0 & 0 & 0 & 0 & 1 \\
& 3 & 3 & 3 & 0 & 0 & 0 & 0 & 0 & 0 & 1 & 1 & 1 & 0 & 0 & 0 & 1 \\
\hline & & & & 0 & 0 & 0 & 0 & 0 & 0 & 0 & 0 & 0 & 1 & 1 & 1 & 1 \\
\hline
\end{tabular}

Note that the diagonal block of order $6 \times 4$ in the matrix of the left hand is the incidence matrix of the affine plane $A G(2,2)$. And the diagonal block of order $12 \times 9$ in the matrix of the right hand is the incidence matrix of the affine plane $A G(2,3)$.

When $q=3$, Latin square $\Sigma^{1}$ is obtained by changing 3 for 0 in $\Sigma_{3}^{1}$, and (2]] $I_{3}$ is obtained from (3]] $I_{3}$ by deleting in the diagonal entries the symbol 3 . The incidence matrix $\left[M \mid \mathcal{P}\left(O_{2,3}\right)^{\top}\right]$ corresponding to item (iii) of Theorem 3.1 is shown next, and gives the incidence matrix of a 3-regular bipartite graph of girth 6 with 8 vertices in each partite set. 


\begin{tabular}{|c|ccc||ccc|ccc||cc|}
\hline \multirow{*}{*}{$\Sigma^{1}$} & 0 & 1 & 2 & 0 & 1 & 0 & 0 & 0 & 1 & 1 & 0 \\
& 1 & 2 & 0 & 1 & 0 & 0 & 0 & 1 & 0 & 1 & 0 \\
& 2 & 0 & 1 & 0 & 0 & 1 & 1 & 0 & 0 & 1 & 0 \\
\hline \multirow{2}{*}{$(2]] I_{3}$} & 12 & 0 & 0 & 1 & 0 & 0 & 1 & 0 & 0 & 0 & 1 \\
& 0 & 12 & 0 & 0 & 1 & 0 & 0 & 1 & 0 & 0 & 1 \\
& 0 & 0 & 12 & 0 & 0 & 1 & 0 & 0 & 1 & 0 & 1 \\
\hline \multirow{2}{*}{$O_{2,3}$} & 1 & 1 & 1 & 1 & 1 & 1 & 0 & 0 & 0 & 0 & 0 \\
\hline & 2 & 2 & 2 & 0 & 0 & 0 & 1 & 1 & 1 & 0 & 0 \\
\hline
\end{tabular}

Case $q=4$. The Latin squares provided by Theorem 2.2 and the incidence matrix of projective plane $P G(2,4)$ given in (6) are the following:

\begin{tabular}{|c|c|c|c|c|c|c|c|c|c|c|c|c|c|c|c|c|c|c|c|c|c|c|c|c|}
\hline \multirow{4}{*}{$\Sigma_{4}^{1}$} & 4 & 1 & 2 & 3 & 0 & & 0 & & & 0 & 1 & & 0 & 0 & $\overline{0}$ & 1 & 1 & 0 & 0 & & $\begin{array}{|ll|}1 & 0\end{array}$ & 0 & 0 & \\
\hline & 1 & 4 & 3 & 2 & 1 & 0 & 0 & 0 & 0 & 0 & 0 & 1 & 0 & 0 & 1 & 0 & 0 & 1 & 0 & 0 & 10 & 0 & 0 & 0 \\
\hline & 2 & 3 & 4 & 1 & 0 & 0 & 0 & 1 & 1 & 0 & 0 & 0 & 0 & 1 & 0 & 0 & 0 & 0 & 1 & 0 & 10 & 0 & 0 & 0 \\
\hline & 3 & 2 & 1 & 4 & 0 & 0 & 1 & 0 & 0 & 1 & 0 & 0 & 1 & 0 & 0 & 0 & 0 & 0 & 0 & 1 & 10 & 0 & 0 & 0 \\
\hline \multirow{4}{*}{$\Sigma_{4}^{2}$} & 4 & 2 & 3 & 1 & $\overline{0}$ & $\overline{0}$ & $\overline{0}$ & 1 & $\overline{0}$ & 1 & 0 & 0 & $\overline{0}$ & $\overline{0}$ & 1 & $\overline{0}$ & 1 & $\overline{0}$ & $\overline{0}$ & $\overline{0}$ & $\begin{array}{|ll|}0 & 1\end{array}$ & 0 & 0 & 0 \\
\hline & 2 & 4 & 1 & 3 & 0 & 0 & 1 & 0 & 1 & 0 & 0 & 0 & 0 & 0 & 0 & 1 & 0 & 1 & 0 & 0 & $\begin{array}{ll}0 & 1\end{array}$ & 0 & 0 & 0 \\
\hline & 3 & 1 & 4 & 2 & 0 & 1 & 0 & 0 & 0 & 0 & 0 & 1 & 1 & 0 & 0 & 0 & 0 & 0 & 1 & 0 & $\begin{array}{ll}0 & 1\end{array}$ & 0 & 0 & 0 \\
\hline & 1 & 3 & 2 & 4 & 1 & 0 & 0 & 0 & 0 & 0 & 1 & 0 & 0 & 1 & 0 & 0 & 0 & 0 & 0 & 1 & $\begin{array}{ll}0 & 1\end{array}$ & 0 & 0 & 0 \\
\hline \multirow{4}{*}{$\Sigma_{4}^{3}$} & 4 & 3 & 1 & 2 & 0 & 0 & 1 & 0 & 0 & 0 & 0 & 1 & 0 & 1 & 0 & 0 & 1 & 0 & 0 & 0 & $\begin{array}{|ll|}0 & 0\end{array}$ & 1 & 0 & 0 \\
\hline & 3 & 4 & 2 & 1 & 0 & 0 & 0 & 1 & 0 & 0 & 1 & 0 & 1 & 0 & 0 & 0 & 0 & 1 & 0 & 0 & $\begin{array}{ll}0 & 0\end{array}$ & 1 & 0 & 0 \\
\hline & 1 & 2 & 4 & 3 & 1 & 0 & 0 & 0 & 0 & 1 & 0 & 0 & 0 & 0 & 0 & 1 & 0 & 0 & 1 & 0 & $\begin{array}{ll}0 & 0\end{array}$ & 1 & 0 & \\
\hline & 2 & 1 & 3 & 4 & 0 & 1 & 0 & 0 & 1 & 0 & 0 & 0 & 0 & 0 & 1 & 0 & 0 & 0 & 0 & 1 & $\begin{array}{ll}0 & 0 \\
\end{array}$ & 1 & 0 & 0 \\
\hline \multirow{4}{*}{$(4]] I_{4}$} & 1234 & 0 & 0 & 0 & 1 & 0 & $\overline{0}$ & $\overline{0}$ & 1 & 0 & 0 & 0 & 1 & 0 & $\overline{0}$ & $\overline{0}$ & 1 & $\overline{0}$ & 0 & $\overline{0}$ & $\begin{array}{|ll|}0 & 0\end{array}$ & 0 & 1 & 0 \\
\hline & 0 & 1234 & 0 & 0 & 0 & 1 & 0 & 0 & 0 & 1 & 0 & 0 & 0 & 1 & 0 & 0 & 0 & 1 & 0 & 0 & $\begin{array}{ll}0 & 0\end{array}$ & 0 & 1 & 0 \\
\hline & 0 & 0 & 1234 & 0 & 0 & 0 & 1 & 0 & 0 & 0 & 1 & 0 & 0 & 0 & 1 & 0 & 0 & 0 & 1 & 0 & $\begin{array}{ll}0 & 0\end{array}$ & 0 & 1 & 0 \\
\hline & 0 & 0 & 0 & 1234 & 0 & 0 & 0 & 1 & 0 & 0 & 0 & 1 & 0 & 0 & 0 & 1 & 0 & 0 & 0 & 1 & $\begin{array}{ll}0 & 0\end{array}$ & 0 & 1 & 0 \\
\hline \multirow{5}{*}{$\mathrm{O}_{4}$} & 1 & 1 & 1 & 1 & 1 & 1 & 1 & 1 & 0 & 0 & 0 & 0 & 0 & 0 & 0 & 0 & $\overline{0}$ & 0 & 0 & 0 & $\begin{array}{|ll|}0 & 0\end{array}$ & 0 & 0 & 1 \\
\hline & 2 & 2 & 2 & 2 & 0 & 0 & 0 & 0 & 1 & 1 & 1 & 1 & 0 & 0 & 0 & 0 & 0 & 0 & 0 & 0 & $\begin{array}{ll}0 & 0\end{array}$ & 0 & 0 & 1 \\
\hline & 3 & 3 & 3 & 3 & 0 & 0 & 0 & 0 & 0 & 0 & 0 & 0 & 1 & 1 & 1 & 1 & 0 & 0 & 0 & 0 & $\begin{array}{lll}0 & 0\end{array}$ & 0 & 0 & 1 \\
\hline & 4 & 4 & 4 & 4 & 0 & 0 & 0 & & 0 & 0 & 0 & 0 & 0 & 0 & 0 & 0 & 1 & 1 & 1 & 1 & $\begin{array}{ll}0 & 0\end{array}$ & 0 & 0 & 1 \\
\hline & 0 & 0 & 0 & 0 & 0 & 0 & 0 & 0 & 0 & 0 & 0 & 0 & 0 & 0 & $\overline{0}$ & & $\overline{0}$ & 0 & 0 & & \begin{tabular}{ll|}
1 & 1
\end{tabular} & 1 & 1 & 1 \\
\hline
\end{tabular}

The diagonal block of order $20 \times 16$ is the incidence matrix of the affine plane $A G(2,4)$.

The incidence matrix $\left[M \mid \mathcal{P}\left(O_{3,4}\right)^{\top}\right]$ corresponding to item (iii) of Theorem 3.1 is shown next, and gives the incidence matrix of a 4-regular bipartite graph of girth 6 with 15 vertices in each partite set.

\begin{tabular}{|c|c|c|c|c|c|c|c|c|c|c|c|c|c|c|c|c|}
\hline \multirow{4}{*}{$\Sigma^{1}$} & 0 & 1 & 2 & 3 & & 1 & $\begin{array}{ll}0 & 0\end{array}$ & 0 & 10 & 1 & 0 & 0 & 0 & 0 & & $\begin{array}{lll}10 & 0\end{array}$ \\
\hline & 1 & 0 & 3 & 2 & 1 & 0 & $\begin{array}{ll}0 & 0\end{array}$ & 0 & 0 & 0 & 1 & 0 & 0 & 10 & & 100 \\
\hline & 2 & 3 & 0 & 1 & 0 & 0 & $\begin{array}{ll}0 & 1\end{array}$ & 1 & 0 & 0 & 0 & 0 & 1 & 0 & & 100 \\
\hline & 3 & 2 & 1 & 0 & 0 & 0 & 10 & 0 & 1 & 0 & 0 & 1 & 0 & 00 & & 100 \\
\hline \multirow{4}{*}{$\Sigma^{2}$} & 0 & 2 & 3 & 7 & 0 & 0 & $\begin{array}{ll}01 \\
\end{array}$ & 10 & 1 & 0 & 0 & 0 & 0 & $\overline{110}$ & & $\begin{array}{lll}0 & 1 & 0\end{array}$ \\
\hline & 2 & 0 & 1 & 3 & 0 & 0 & 10 & 1 & 0 & 0 & 0 & 0 & 0 & 01 & & $\begin{array}{lll}0 & 1 & 0\end{array}$ \\
\hline & 3 & 1 & 0 & 2 & 0 & 1 & $\begin{array}{ll}0 & 0\end{array}$ & 0 & 0 & 0 & 1 & 1 & 0 & 0 & & $\begin{array}{llll}0 & 1 & 0\end{array}$ \\
\hline & 1 & 3 & 2 & 0 & 1 & 0 & $\begin{array}{ll}0 & 0 \\
\end{array}$ & 0 & 0 & 1 & 0 & 0 & 1 & $0 \quad 0$ & & $\begin{array}{llll}0 & 1 & 0\end{array}$ \\
\hline \multirow{4}{*}{ (3]]] $I_{4}$} & 123 & 0 & 0 & 0 & 1 & 0 & 0 & 1 & 0 & 0 & 0 & 1 & 0 & $0 \quad 0$ & & $\begin{array}{lll}0 & 0 & 1\end{array}$ \\
\hline & 0 & 123 & 0 & 0 & 0 & 1 & $\begin{array}{ll}0 & 0\end{array}$ & 0 & 1 & 0 & 0 & 0 & 1 & 0 & & $\begin{array}{lll}0 & 0 & 1\end{array}$ \\
\hline & 0 & 0 & 123 & 0 & 0 & 0 & 10 & ) & 0 & 1 & 0 & 0 & 0 & 10 & & $\begin{array}{lll}0 & 0 & 1\end{array}$ \\
\hline & 0 & 0 & 0 & 123 & 0 & 0 & $\begin{array}{ll}0 & 1\end{array}$ & 0 & 0 & 0 & 1 & 0 & 0 & 01 & 1 & $\begin{array}{lll}0 & 0 & 1\end{array}$ \\
\hline \multirow{3}{*}{$O_{3,4}$} & 1 & 1 & 1 & 1 & 1 & 1 & $\overline{11}$ & 70 & 10 & $\overline{0}$ & 0 & 0 & 0 & $\overline{010}$ & & $\begin{array}{llll}0 & 0 & 0\end{array}$ \\
\hline & 2 & 2 & 2 & 2 & 0 & 0 & $\begin{array}{ll}0 & 0\end{array}$ & 1 & 1 & 1 & 1 & 0 & 0 & $0 \quad 0$ & & $\begin{array}{lll}0 & 0 & 0\end{array}$ \\
\hline & 3 & 3 & 3 & 3 & 0 & 0 & & ) & 0 & 0 & 0 & 1 & 1 & & & $\begin{array}{lll}0 & 0 & 0\end{array}$ \\
\hline
\end{tabular}


To give a method for constructing incidence matrices of $(q-r)$-regular balanced bipartite graphs, $1 \leq r \leq q-3$, of girth 6 with $2\left(q^{2}-r q-1\right)$ vertices we need the following notation. Let $\Sigma_{r}^{u}$ be the matrix obtained from $\Sigma^{u}$ by replacing every $q-i(i=1, \ldots, r)$ with 0 except the value $q-r$ which remains in the first $r$ columns. Let $\Sigma_{r *}^{q-r-1}$ be the matrix of order $r \times q$ consisting of the $r$ rows of $\Sigma_{r}^{q-r-1}$ having an entry equal to $q-r$; and let $O_{q-r-1, q}^{*}$ be the matrix obtained from $O_{q-r-1, q}$ by changing the entries $\left(O_{q-r-1, q}\right)_{s t}=s$ for zero in every column $t$ such that $\left(\Sigma_{r *}^{q-r-1}\right)_{h t}=s, h=1, \ldots, r$. For instance, suppose $q=5$ and $r=2$. Then

$$
\left.\left.\Sigma_{2}^{1}=\left[\begin{array}{lllll}
0 & 1 & 2 & 0 & 0 \\
1 & 2 & 0 & 0 & 0 \\
2 & 3 & 0 & 0 & 1 \\
3 & 0 & 0 & 1 & 2 \\
0 & 0 & 1 & 2 & 0
\end{array}\right] \quad \Sigma_{2}^{2}=\left[\begin{array}{lllll}
0 & 2 & 0 & 1 & 0 \\
2 & 0 & 1 & 0 & 0 \\
0 & 1 & 0 & 0 & 2 \\
1 & 3 & 0 & 2 & 0 \\
3 & 0 & 2 & 0 & 1
\end{array}\right] \quad \Sigma_{2^{*}}^{2}=\begin{array}{lllll}
1 & 3 & 0 & 2 & 0 \\
3 & 0 & 2 & 0 & 1
\end{array}\right] \quad O_{2,5}^{*}=\begin{array}{llllll}
0 & 1 & 1 & 1 & 0 \\
2 & 2 & 0 & 0 & 2
\end{array}\right]
$$

Moreover, let $(q-r]] I_{q}^{*}$ be the matrix obtained from $\left.(q-r]\right] I_{q}$ by deleting the symbol $q-r$ from the entry-sets in the last $q-r-1$ columns and by adding the symbol $q-r$ in all the places $(i, r+1), i=r+2, \ldots, q$. And let $O_{*}$ be the matrix obtained from $O_{q-r-2, q}$ by changing the entries $\left(O_{q-r-2, q}\right)_{u t}=u$ for zero in every column $t$ such that in the row $t$ of $\Sigma_{r}^{u}$ has a symbol $q-r$, i.e., $\left(\Sigma_{r}^{u}\right)_{t h}=q-r, h=1, \ldots, r$. Thus for $q=5$ and $r=2$ we have

$$
\left.(3]] I_{5}^{*}=\begin{array}{lllll}
123 & 0 & 0 & 0 & 0 \\
0 & 123 & 0 & 0 & 0 \\
0 & 0 & 123 & 0 & 0 \\
0 & 0 & 3 & 12 & 0 \\
0 & 0 & 3 & 0 & 12
\end{array}\right] \quad O_{*}=\begin{array}{lllll}
1 & 1 & 0 & 0 & 1 \\
0
\end{array}
$$

Theorem 3.2 Let $q \geq 4$ be a prime power and $\mathbb{F}_{q}$ a finite Galois field of $q$ elements, and let $r$ be an integer such that $1 \leq r \leq q-3$. Let $M$ be the position matrix of $\left\{\Sigma_{r}^{1}, \ldots, \Sigma_{r}^{q-r-2}, \Sigma_{r *}^{q-r-1},(q-\right.$ $\left.r]] I_{q}^{*}, O_{q-r-1, q}^{*}\right\}$ and let $M^{\prime}$ be the matrix obtained from $M$ once eliminated the columns full of zeros. Then $\left[M^{\prime} \mid \mathcal{P}\left(O_{*}\right)^{\top}\right]$ is the incidence matrix of a $(q-r)$-regular bipartite graph of girth 6 and $q^{2}-r q-1$ vertices in each partite set.

Proof: Note that $\left[M \mid \mathcal{P}\left(O^{*}\right)^{\top}\right]$ is a matrix free of $J_{2}$ because it is a submatrix of (6) by changing some ones for zeros. Besides, $M$ has order $\left(q^{2}-r q-1\right) \times q(q-r)$, and the last $q-r-1$ columns of $M$ are full of zeros, because in the last $q-r-1$ columns of each $\Sigma_{r}^{u}$ we have replaced the entry $q-r$ with zero. Thus, we consider the matrix $M^{\prime}$ once eliminated from $M$ these columns full of zeros. Hence $M^{\prime}$ has order $\left(q^{2}-r q-1\right) \times(q(q-r)-(q-r-1))$. Furthermore, notice that each of the $q^{2}-r q-q+r+1$ columns of $M^{\prime}$ has $q-r$ ones according to the definition of $M^{\prime}$. On the other hand, the rows of the position matrix corresponding to $\left.\left\{\Sigma_{r *}^{q-r-1},(q-r]\right] I_{q}^{*}, O_{q-r-1, q}^{*}\right\}$ have $q-r$ ones each because of the definition of theses matrices. Therefore, since the value $q-r$ remains in the first $r$ columns of each $\Sigma_{r}^{u}, u=1, \ldots, q-r-2$, then there are $(q-r-2)(q-r)$ rows in $M^{\prime}$ having $q-r-1$ ones, and the remaining rows have $q-r$ ones each. By joining $\mathcal{P}\left(O_{*}\right)^{\top}$ to $M^{\prime}$ we put $q-r-2$ more columns, that due to the definition of $O_{*}$ add an additional one just in the rows which had $q-r-1$ ones in $M^{\prime}$. Therefore $\left[M^{\prime} \mid \mathcal{P}\left(O_{*}\right)^{\top}\right]$ is the incidence matrix of a $(q-r)$-regular bipartite graph with $q^{2}-r q-1$ vertices in each partite set and girth at least 6 . Since the number of vertices of the constructed graphs is strictly less than the lower bound given in (1) for $g=8$, then the girth is 6 . 
To illustrate the method of Theorem 3.2 let us consider the first cases $q=4,5$.

Case $q=4$ and $r=1$. The matrices of Theorem 3.2 and the incidence matrix of a 3-regular bipartite graph of girth 6 with 11 vertices in each partite set are the following:

\begin{tabular}{|c|cccc||cccc|cccc|cccc|c|}
\hline \multirow{5}{*}{$\Sigma_{1}^{1}$} & 0 & 1 & 2 & 0 \\
& 1 & 0 & 0 & 2 & 0 & 1 & 0 & 0 & 0 & 0 & 1 & 0 & 0 & 0 & 0 & 0 & 1 \\
& 2 & 0 & 0 & 0 & 0 & 0 & 0 & 0 & 1 & 0 & 0 & 0 & 0 & 1 \\
& 3 & 2 & 1 & 0 & 0 & 0 & 1 & 1 & 0 & 0 & 0 & 0 & 0 & 0 & 0 & 1 \\
0 & 0 & 1 & 0 & 0 & 1 & 0 & 0 & 1 & 0 & 0 & 0 & 0 \\
\hline$\Sigma_{1 *}^{2}$ & 3 & 1 & 0 & 2 & 0 & 1 & 0 & 0 & 0 & 0 & 0 & 1 & 1 & 0 & 0 & 0 & 0 \\
\hline \multirow{3}{*}{$(3]] I_{4}^{*}$} & 123 & 0 & 0 & 0 & 1 & 0 & 0 & 0 & 1 & 0 & 0 & 0 & 1 & 0 & 0 & 0 & 0 \\
& 0 & 123 & 0 & 0 & 0 & 1 & 0 & 0 & 0 & 1 & 0 & 0 & 0 & 1 & 0 & 0 & 0 \\
& 0 & 3 & 12 & 0 & 0 & 0 & 1 & 0 & 0 & 0 & 1 & 0 & 0 & 1 & 0 & 0 & 0 \\
& 0 & 3 & 0 & 12 & 0 & 0 & 0 & 1 & 0 & 0 & 0 & 1 & 0 & 1 & 0 & 0 & 0 \\
\hline \multirow{3}{*}{$O_{2,4}^{*}$} & 1 & 0 & 1 & 1 & 1 & 0 & 1 & 1 & 0 & 0 & 0 & 0 & 0 & 0 & 0 & 0 & 0 \\
& 2 & 2 & 2 & 0 & 0 & 0 & 0 & 0 & 1 & 1 & 1 & 0 & 0 & 0 & 0 & 0 & 0 \\
\hline
\end{tabular}

The matrix $M^{\prime}$ of Theorem 3.2 is obtained by deleting the columns 11 and 12 full of zeros.

Case $q=5$ and $r=2$. The matrices of Theorem 3.2 and the incidence matrix of a 3-regular bipartite graph of girth 6 with 14 vertices in each partite set are the following:

\begin{tabular}{|c|ccccc||ccccc|ccccc|ccccc|c|}
\hline \multirow{5}{*}{$\Sigma_{2}^{1}$} & 0 & 1 & 2 & 0 & 0 & 0 & 1 & 0 & 0 & 0 & 0 & 0 & 1 & 0 & 0 & 0 & 0 & 0 & 0 & 0 & 1 \\
& 1 & 2 & 0 & 0 & 0 & 1 & 0 & 0 & 0 & 0 & 0 & 1 & 0 & 0 & 0 & 0 & 0 & 0 & 0 & 0 & 1 \\
& 2 & 3 & 0 & 0 & 1 & 0 & 0 & 0 & 0 & 1 & 1 & 0 & 0 & 0 & 0 & 0 & 1 & 0 & 0 & 0 & 0 \\
& 3 & 0 & 0 & 1 & 2 & 0 & 0 & 0 & 1 & 0 & 0 & 0 & 0 & 0 & 1 & 1 & 0 & 0 & 0 & 0 & 0 \\
& 0 & 0 & 1 & 2 & 0 & 0 & 0 & 1 & 0 & 0 & 0 & 0 & 0 & 1 & 0 & 0 & 0 & 0 & 0 & 0 & 1 \\
\hline$\Sigma_{2^{*}}^{2}$ & 1 & 3 & 0 & 2 & 0 & 1 & 0 & 0 & 0 & 0 & 0 & 0 & 0 & 1 & 0 & 0 & 1 & 0 & 0 & 0 & 0 \\
& 3 & 0 & 2 & 0 & 1 & 0 & 0 & 0 & 0 & 1 & 0 & 0 & 1 & 0 & 0 & 1 & 0 & 0 & 0 & 0 & 0 \\
\hline \multirow{3}{*}{$(3]] I_{5}^{*}$} & 123 & 0 & 0 & 0 & 0 & 1 & 0 & 0 & 0 & 0 & 1 & 0 & 0 & 0 & 0 & 1 & 0 & 0 & 0 & 0 & 0 \\
& 0 & 123 & 0 & 0 & 0 & 0 & 1 & 0 & 0 & 0 & 0 & 1 & 0 & 0 & 0 & 0 & 1 & 0 & 0 & 0 & 0 \\
& 0 & 0 & 123 & 0 & 0 & 0 & 0 & 1 & 0 & 0 & 0 & 0 & 1 & 0 & 0 & 0 & 0 & 1 & 0 & 0 & 0 \\
& 0 & 0 & 3 & 12 & 0 & 0 & 0 & 0 & 1 & 0 & 0 & 0 & 0 & 1 & 0 & 0 & 0 & 1 & 0 & 0 & 0 \\
& 0 & 0 & 3 & 0 & 12 & 0 & 0 & 0 & 0 & 1 & 0 & 0 & 0 & 0 & 1 & 0 & 0 & 1 & 0 & 0 & 0 \\
\hline \multirow{3}{*}{$O_{2,5}^{*}$} & 0 & 1 & 1 & 1 & 0 & 0 & 1 & 1 & 1 & 0 & 0 & 0 & 0 & 0 & 0 & 0 & 0 & 0 & 0 & 0 & 0 \\
& 2 & 2 & 0 & 0 & 2 & 0 & 0 & 0 & 0 & 0 & 1 & 1 & 0 & 0 & 1 & 0 & 0 & 0 & 0 & 0 & 0 \\
\hline
\end{tabular}

The matrix $M^{\prime}$ of Theorem 3.2 is obtained by deleting the columns 14 and 15 full of zeros.

\section{Improvement for $(q-1)$-regular bipartite graphs of girth 6}

In this section we improve Theorem 3.2 for $r=1$, finding incidence matrices of $(q-1)$-regular bipartite graphs of girth 6 with $q^{2}-q-2$ vertices in each partite set. To this end we need to introduce the following definition.

Definition 4.1 Two $C_{4}$-free matrices $A^{1}$ and $A^{2}$ of the same number of columns whose elements are 1-sets of $[[n]]$ are said to be quasi row-disjoint if and only if the cartesian product of any two rows $\left(A^{1}\right)_{i},\left(A^{2}\right)_{h}$ contains at most one pair $(x, x) \in\left(A^{1}\right)_{i} \times\left(A^{2}\right)_{h}$ with $x \neq 0$. 
An example of two quasi row-disjoint Latin squares of order $3 \times 3$, one with entries in (3]] and the other with entries in $[[3]] \backslash\{1\}$ is the following.

$$
L^{1}=\left(\begin{array}{lll}
1 & 2 & 3 \\
2 & 3 & 1 \\
3 & 1 & 2
\end{array}\right) \quad L^{2}=\left(\begin{array}{lll}
0 & 3 & 2 \\
3 & 2 & 0 \\
2 & 0 & 3
\end{array}\right)
$$

We can check by superimposing any two rows of $L^{1}$ and $L^{2}$, that there is at most one resulting pair $(x, x)$ with $x \neq 0$. Then $L^{1}$ and $L^{2}$ are quasi row-disjoint.

Theorem 4.1 Let $Q^{1}$ and $Q^{2}$ be two $C_{4}$-free matrices of the same number of columns and whose elements are 1-sets of $[[n]]$. Then $Q^{1}$ and $Q^{2}$ are quasi row-disjoint if and only the family $\left\{Q^{1}, Q^{2}\right\}$ is $C_{4}$-free.

Proof: Let $\left(Q^{\ell}\right)_{i}$ denote the row $i$ of $Q^{\ell}, \ell=1,2$. Assume that $(x, x) \in\left(Q^{1}\right)_{i} \times\left(Q^{2}\right)_{h}$ with $x \in[[n]], x \neq 0$. Then $x=\left(Q^{1}\right)_{i j}=\left(Q^{2}\right)_{h j}$ or, equivalently $\left(P_{x}\left(Q^{1}\right)\right)_{i j}=\left(P_{x}\left(Q^{2}\right)\right)_{h j}=1$, for some column $j$, where $P_{x}\left(Q^{\ell}\right)$ is the position matrix of $x$ in $Q^{\ell}$.

If $Q^{1}$ and $Q^{2}$ are not quasi row-disjoint, then there exists $(y, y) \in\left(Q^{1}\right)_{i} \times\left(Q^{2}\right)_{h}$ with $y \in[[n]] \backslash\{x, 0\}$. This implies that $\left(P_{y}\left(Q^{1}\right)\right)_{i k}=\left(P_{y}\left(Q^{2}\right)\right)_{h k}=1$ with $k \neq j$, and therefore the position matrix of $\left\{Q^{1}, Q^{2}\right\}$ contains a submatrix $J_{2}$, i.e., $\left\{Q^{1}, Q^{2}\right\}$ is not $C_{4}$-free.

Conversely, if the position matrix of $\left\{Q^{1}, Q^{2}\right\}$ contains $J_{2}$ as a submatrix, then there exists $y \in[[n]] \backslash\{x, 0\}$ such that $\left(P_{y}\left(Q^{1}\right)\right)_{i k}=\left(P_{y}\left(Q^{2}\right)\right)_{h k}=1$, or in other words, $y=\left(Q^{1}\right)_{i k}=\left(Q^{2}\right)_{h k}$, $k \neq j$. Hence $Q^{1}, Q^{2}$ are not quasi row-disjoint.

As a consequence of Theorems 2.2, Remark 2.1 and Theorem 4.1, the following corollary is now clear.

Corollary 4.1 Let $q$ be a prime power and $\mathbb{F}_{q}$ the Galois field of order $q$. The family $\left\{\Sigma_{q}^{u}: u \in\right.$ $\left.\left.\mathbb{F}_{q}\right\} \cup\{(q]] I_{q}, O_{q}\right\}$ is a set of $q+1$ quasi row-disjoint matrices of order $q$ with entries in [[q]].

Next, we construct a family of mutually quasi row-disjoint matrices of order $q-1$ by adding to each entry of the product table of the cyclic group $\left(\mathbb{F}_{q} \backslash\{0\}, \cdot\right)$ an element of the Galois field $\mathbb{F}_{q}$.

Theorem 4.2 Let $q \geq 4$ be a prime power and $\mathbb{F}_{q}$ the Galois field of order $q$. Then the following assertions are true.

(i) For every $u \in \mathbb{F}_{q}$ define the matrix $\Pi^{u}$ by $\left(\Pi^{u}\right)_{i j}=u+i j, i, j \in \mathbb{F}_{q}, i, j \neq 0$. Then the family $\left\{\Pi^{u}: u \in \mathbb{F}_{q}\right\}$ is $C_{4}$-free. 
(ii) For every $u \in \mathbb{F}_{q}$ define the matrix $D^{u}$ by $\left(D^{u}\right)_{i j}=u-i j, i, j \in \mathbb{F}_{q}, i, j \neq 0$. Then the family $\left\{D^{u}: u \in \mathbb{F}_{q}\right\}$ is $C_{4}$-free.

(iii) If $N$ is the position matrix of $\left\{\Pi^{u}: u \in \mathbb{F}_{q}, u \neq 0\right\}$, then the transpose of $N$ is the position matrix of $\left\{D^{u}: u \in \mathbb{F}_{q}, u \neq 0\right\}$.

(iv) Let $N$ be the position matrix of $\left.\left\{\Pi^{3}, \ldots, \Pi^{q-1}, \Pi^{0},(q-1]\right] I_{q-1}, O_{q-1}^{2}\right\}$, where $O_{q-1}^{2}$ is the matrix obtained from $O_{q-1}$ by deleting the first two rows. Then the $(0,1)$-matrix $\left[N \mid \mathcal{P}\left(O_{q-3, q-1}\right)^{\top}\right]$ is the incidence matrix of a $(q-1)$-regular bipartite graph of girth 6 and $q^{2}-q-2$ vertices in each partite set.

Proof: $(i)$ Clearly $\left\{\Pi^{u}=[i j+u]: u, i, j \in \mathbb{F}_{q}, i, j \neq 0\right\}$ is a set of $q$ Latin squares of order $q-1$, each one with entries from $[[q-1]] \backslash\{u\}$, because $i j+u=u$ implies $i=0$ or $j=0$ which is impossible by definition of $\Pi^{u}$. Let us see that they are mutually quasi row-disjoint. Suppose $\left(\Pi^{w}\right)_{i j}=\left(\Pi^{u}\right)_{k j}$ for some $u, w \in \mathbb{F}_{q}$ with $u \neq w$ and $i, j, k \neq 0$. Therefore

$$
i j+w=k j+u \text {. }
$$

If the Latin squares are not quasi row-disjoint, then there exists $h \neq j, h \neq 0$, such that $\left(\Pi^{w}\right)_{i h}=\left(\Pi^{u}\right)_{k h}$, that is,

$$
i h+w=k h+u .
$$

Hence $i(j-h)=k(j-h)$. As $j \neq h$, then $j-h$ has an inverse respect to the product in the cyclic group $\mathbb{F}_{q} \backslash\{0\}$ yielding $i=k$, thus $u=w$ which is a contradiction. Therefore $\Pi^{u}$ and $\Pi^{w}$ are quasi row-disjoint, and hence by Theorem 4.1 we conclude that $\left\{\Pi^{u}: u \in \mathbb{F}_{q}\right\}$ is $C_{4}$-free.

(ii) The proof of this item is the same as the proof of item $(i)$.

(iii) Reasoning as in the proof of item (iii) of Theorem 2.2, it is enough to prove that $P_{k}\left(\Pi^{w}\right)=P_{w}\left(D^{k}\right)$ for all $k, w \in \mathbb{F}_{q}, k \neq 0$. Since $\left(P_{k}\left(\Pi^{w}\right)\right)_{i j}=1$ if and only if $w+i j=k$, hence $w=k-i j$ if and only if $\left(P_{w}\left(D^{k}\right)\right)_{i j}=1$. Then $(i i i)$ is valid.

(iv) By point $(i)$ we know that $\left\{\Pi^{u}: u \in \mathbb{F}_{q}\right\}$ is $C_{4}$-free. It is not difficult to see that $O_{q-1}$ is quasi row-disjoint with each $\Pi^{u}$, and using Remark 2.1 we obtain that $\left\{\Pi^{u}: u \in\right.$ $\left.\left.\mathbb{F}_{q}\right\} \cup\{(q-1]] I_{q-1}, O_{q-1}\right\}$ is a $C_{4}$-free family of of $q+2$ mutually quasi row-disjoint $C_{4}$-free matrices of order $(q-1) \times(q-1)$ whose entries are subsets of $[[q-1]]$. Therefore the position matrix $N$ of $\left.\left\{\Pi^{3}, \ldots, \Pi^{q-1}, \Pi^{0},(q-1]\right] I_{q-1}, O_{q-1}^{2}\right\}$ is free of $J_{2}$.

Moreover, we have for all $u \in \mathbb{F}_{q}$ that the entries of $\Pi^{u}$ belong to $[[q-1]] \backslash\{u\}$. Hence the rows of the position matrix of each $\Pi^{u}$ have $q-2$ ones for $u \neq 0$, and $q-1$ ones for $u=0$. Let $A$ be the position matrix of $\left\{\Pi^{1}, \ldots, \Pi^{q-1}\right\}$. From item (iii) it follows that $A^{\top}$ is the position matrix of $\left\{D^{1}, \ldots, D^{q-1}\right\}$. This implies that the columns of matrix $A$ have $q-2$ ones. Then deleting the matrices $\Pi^{1}$ and $\Pi^{2}$ results a submatrix $A^{\prime}$ of $A$ which has $q-3$ ones in $2(q-1)$ columns and $q-4$ ones in the $(q-3)(q-1)$ remaining columns. Let $B$ be the position matrix of $\left.\left\{\Pi^{0},(q-1]\right] I_{q-1}\right\}$ which is readily seen that has 2 ones in each column and $q-1$ ones in each row. Therefore joining to $A^{\prime}$ the rows of $B$ we obtain a matrix $C$ which has $q-1$ ones in $2(q-1)$ columns and $q-2$ ones in the $(q-3)(q-1)$ remaining columns. Finally, adding to 
$C$ the rows of the position matrix of $O_{q-1}^{2}$ we obtain the matrix $N$ having $q-1$ ones in each column. Thus, $\left[N \mid \mathcal{P}\left(O_{q-3, q-1}\right)^{\top}\right]$ is the incidence matrix of a $(q-1)$-regular bipartite graph of girth 6 and $q^{2}-q-2$ vertices in each partite set.

To illustrate the method contained in Theorem 4.2, let us consider the case $q=4$.

Case $q=4$. The $C_{4}$-free quasi row-disjoint matrices provided by Theorem 4.2 and the incidence matrix of a 3-regular bipartite graph of girth 6 with 10 vertices in each partite set are the following:

\begin{tabular}{|c|ccc||ccc|ccc|ccc|c|}
\hline \multirow{3}{*}{$\Pi^{3}$} & 2 & 1 & 0 & 0 & 1 & 0 & 1 & 0 & 0 & 0 & 0 & 0 & 1 \\
& 1 & 0 & 2 & 1 & 0 & 0 & 0 & 0 & 1 & 0 & 0 & 0 & 1 \\
& 0 & 2 & 1 & 0 & 0 & 1 & 0 & 1 & 0 & 0 & 0 & 0 & 1 \\
\hline \multirow{3}{*}{$\Pi^{0}$} & 1 & 2 & 3 & 1 & 0 & 0 & 0 & 1 & 0 & 0 & 0 & 1 & 0 \\
& 2 & 3 & 1 & 0 & 0 & 1 & 1 & 0 & 0 & 0 & 1 & 0 & 0 \\
& 3 & 1 & 2 & 0 & 1 & 0 & 0 & 0 & 1 & 1 & 0 & 0 & 0 \\
\hline \multirow{3}{*}{$(3]] I_{3}$} & 123 & 0 & 0 & 1 & 0 & 0 & 1 & 0 & 0 & 1 & 0 & 0 & 0 \\
& 0 & 0 & 123 & 0 & 1 & 0 & 0 & 1 & 0 & 0 & 1 & 0 & 0 \\
& 0 & 0 & 1 & 0 & 0 & 1 & 0 & 0 & 1 & 0 \\
\hline$O_{3}^{2}$ & 3 & 3 & 3 & 0 & 0 & 0 & 0 & 0 & 0 & 1 & 1 & 1 & 0 \\
\hline
\end{tabular}

\section{Conclusions}

We have presented a method providing the incidence matrices of both affine planes and projective planes of order $q$, and $q$-regular bipartite graphs with $q^{2}-1$ vertices in each partite set when $q$ is a prime power. From these incidence matrices, we obtain the incidence matrices of $(q-r)$ regular bipartite graphs of girth 6 having $q^{2}-r q-1$ vertices in each partite set. Furthermore, using quasi row-disjoint Latin squares we improve this result for $r=1$ finding $(q-1)$-regular bipartite graphs of girth 6 with $q^{2}-q-2$ vertices in each partite set. Table 1 shows a comparison between the number of vertices of some known smallest graphs $[1,20]$, and the graphs provided by the method proved in this work. We only consider degrees $k$ in the interval $[q-r, q+1]$ in which the only prime power is $q$, and $q-r$ is different from a prime power plus one.

\section{Acknowledgments}

We would like to express my thanks to the referees for their helpful comments and suggestions. Over all I want to thank to Kevin Phelps for suggesting me that the Latin squares obtained by multiplying each entry of the addition table of the Galois field by an element distinct from zero are the $(1,3)$ conjugates of Latin squares of a complete set of MOLS. 


\begin{tabular}{|c|c|c|}
\hline$k$ & known $(k, 6)$-graph & Method \\
\hline \hline 7 & $90[17]$ & 96 \\
11 & $240[1,22]$ & 240 \\
13 & $336[1]$ & 336 \\
15 & $462[1]$ & 476 \\
16 & $504[1]$ & 510 \\
$q$ prime power & & \\
$q+1$ & $2\left(q^{2}+q+1\right)$ Projective plane & $2\left(q^{2}+q+1\right)$ \\
$q$ & $2\left(q^{2}-1\right)[1]$ & $2\left(q^{2}-1\right)$ \\
$q-1$ & $2\left(q^{2}-q-1\right)[1]$ & $2\left(q^{2}-q-2\right)$ \\
$q-r, r \geq 2$ & $2\left(q^{2}-r q\right)[2]$ & \\
& $2\left(q^{2}-r q-1\right)[1,2]$ & $2\left(q^{2}-r q-1\right)$ \\
\hline \hline
\end{tabular}

Table 1: Order of some (minimal) regular graphs of girth 6 .

\section{References}

[1] M. Abreu, M. Funk, D. Labbate, V. Napolitano, On (minimal) regular graphs of girth 6, Australasian Journal of Combinatorics 35 (2006) pp. 119-132.

[2] G. Araujo, D. González-Moreno, J.J. Montellano, O. Serra, On upper bounds and conectivity of cages. Australasian Journal of Combin. 38: (2007) pp. 221-228.

[3] E. Bannai And T. Ito, On finite Moore graphs, J. Fac. Sci. Univ. Tokio, Sect. I A Math 20 (1973) pp. 191-208.

[4] L.M. Batten, Combinatorics of finite geometries, Cambridge University Press, Cambridge, UK, 1997.

[5] C.T. Benson, Minimal regular graphs of girth eight and twelve, Canad. J. Math 18 (1966), pp. 1091-1094.

[6] N. Biggs, Algebraic Graph Theory, Cambridge University Press, New York, 1996.

[7] N. Biggs, Construction for cubic graphs with large girth, The electronic Journal of combinatorics 5 (1998), \#A1.

[8] G. Brinkmann, B. D. McKay and C. SaAger, The smallest cubic graphs of girth nine, Combinatorics Probability and Computing 5, (1995), pp. 1-13.

[9] A. M. Cohen And J. Tits, On generalized hexagons and a near octagon whose lines have three points, European J. Combin., 6 (1985), pp. 13-27.

[10] P. ERdös And H. Sachs, Reguläre Graphen gegebener Taillenweite mit minimaler Knotenzahl, Wiss. Z. Uni. Halle (Math. Nat.), 12 (1963), pp. 251-257. 
[11] G. Exoo, A Simple Method for Constructing Small Cubic Graphs of Girths 14, 15 and 16. Electronic Journal of Combinatorics, 3, 1996.

[12] W. Feit and G. Higman, The non-existence of certain generalized polygons, J. Algebra 1 (1964) pp. 114-131.

[13] C. Godsil And G. Royle, Algebraic Graph Theory, Springer, NY 2000.

[14] D.A. Holton And J. Sheehan, The Petersen Graph, Chapter 6: Cages, Cambridge University (1993).

[15] J. H. van Lint and R. M. Wilson, A course in Combinatorics, Cambridge University Press, UK 1994.

[16] M. O'Keefe And P.K. Wong, A smallest graph of girth 10 and valency 3, Journal of Combinatorial Theory (B) 29, 1980, pp. 91 - 105.

[17] M. O'Keefe And P.K. Wong, The smallest graph of girth 6 and valency 7, J. Graph Theory 5 (1981), no. 1, pp. 79-85.

[18] M. Meringer, Fast generation of regular graphs and construction of cages, Journal of Graph Theory 30 (1999), pp. 137-146.

[19] T. Pisanski, M. Boben, D. Marusic, A. Orbanic, A. Graovac, The 10-cages and derived configurations, Discrete Mathematics 275 (2004) p. 265-276.

[20] G. RoYLE, http://www.cs.uwa.edu.au/ gordon/data.html.

[21] W. T. Tutte, A family of cubical graphs. Proc. Cambridge Philos. Soc., (1947), pp. 459474 .

[22] P.K. Wong, A regular graph of girth 6 and valency 11 Internat. J. Math. Math. Sci 9, 1986, 561-565.

[23] P. K. Wong, Cages-a survey, Journal of Graph Theory 6 (1982), pp. 1-22. 